\title{
Green Synthesis of Phytochemical Nanoparticles and their Antimicrobial Activity, A Review Study
}

\author{
Aditi Chowdhury, Joushan Ara and Md Shahidul Islam* \\ Department of Pharmacy, University of Science and Technology Chittagong (USTC), Chattogram, Bangladesh \\ *Corresponding author: Md Shahidul Islam, Department of Pharmacy, University of Science and Technology Chittagong \\ (USTC), Chattogram, Bangladesh
}

\section{ARTICLE INFO}

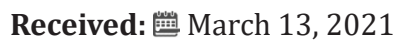

Published: March 18, 2021

Citation: Aditi Chowdhury, Joushan Ara, Md Shahidul Islam. Green Synthesis of Phytochemical Nanoparticles and Their Antimicrobial Activity, A Review Study. Biomed J Sci \& Tech Res 34(4)-2021. BJSTR. MS.ID.005580.

Keywords: Nanoparticles; Review; Green Synthesis; Antimicrobial Activity

\begin{abstract}
Rapid industrialization, urbanization and population explosion are resulting in deterioration of earth atmosphere and a huge amount of hazardous and unwanted substances are being released. It is now high time to learn about the secrets that are present in the nature and its natural products which lead to advancements in the synthesis processes of NPs. Traditionally nanoparticles were produced only by physical and chemical methods. Often, chemical synthesis method leads to presence of some of the toxic chemical absorbed on the surface that may have adverse effect in the medical applications. This is not an issue when it comes to biosynthesized nanoparticles via green synthesis route. So, in the search of cheaper pathways for nanoparticles synthesis, scientist used microbial enzymes and plant extracts like phytochemicals. In this review, Ag metal is used because of its specific properties like larger surface area to volume ratio, less reactive, more bioactive, potent and stable. Different phytochemical compounds of plants such as flavonoids, saponins, alkaloids, phenolic compounds, tannins, terpenoids etc. are mostly bound with Ag+ ions to form complex that give good antimicrobial effect. It is investigated that the zone of inhibition of plant extracts against microorganisms are less than the zone of inhibition of AgNps. So, it can be said that AgNps are more bioactive and potent than the plant extracts and have good antimicrobial activity. Future studies will probably focus on obtaining other nanoparticles with antimicrobial effects at its maximum level and toxicity at minimum.
\end{abstract}

\section{Introduction}

Incorporation of green chemistry techniques and methodologies into nanotechnology is of great interest which has gained much attention over the past decade [1]. Furthermore, NPs are widely applied to human contact areas and there is a growing need to develop processes for synthesis that do not use harsh toxic chemicals [2]. The nanoparticles synthesized from chemical and physical methods generally require high temperature, pressure, expensive equipment, toxic chemicals, and reagents and most importantly capping agents for the stabilization of nanoparticles; thus, these methods are toxic to environment and nonecofriendly [3]. With their antioxidant or reducing properties they are usually responsible for the reduction of metal compounds into their respective nanoparticles [4]. The conventional methods for the production of NPs are expensive, toxic, and non-environment friendly. To overcome these problems, researchers have found the precise green routes like the naturally occurring sources and their products that can be used for the synthesis of NPs [5].

Therefore, green/biological synthesis of NPs is a possible alternative to chemical and physical methods [6]. Biological methods of synthesis have thus paved way for the "greener synthesis" of nanoparticles and these have proven to be better methods due to slower kinetics [7]. Recently, green methods using plant extracts have been developed as an alternative for common chemical and physical methods to synthesize noble metal NPs. Due to the presence of reducing agents like alkaloids, polyphenols, and flavonoids which are major phytoconstituents of the plant extracts, and stabilizing agents such as polysaccharides and proteins, stable metal NPs can be easily synthesized using the plant extracts. Green synthesis provides advancement over chemical and physical method as it is cost effective, environment friendly, easily scaled up for large 
scale synthesis [8]. Green synthesis of nanoparticles has attracted considerable attention in recent years. Several metal nanoparicles such as Cobalt (Co), Copper (Cu), Zinc (Zn), Iron (Fe), Silver (Ag), Lead (Pb), Manganese (Mn), Magnesium (Mg), Palladium (Pd), Gold $\mathrm{Au})$ are used in green synthesis. Among the several noble metal nanoparticles, AgNPs have attracted special attention due to their unique properties including appropriate electrical conductivity, chemical stability, catalytic and antimicrobial activities. Because of high surface to volume ratio, silver in nanoscale has demonstrated completely different properties from bulk particles made from the same material.
Therefore, synthesis of AgNPs is an emerging area and interesting subject. In green synthesis, a solvent (usually water) is chosen and employed in step one. A non-toxic reducing and stabilizer agents are utilized in steps two and three, respectively. In this method, solvents, reducing, and stabilizers agents are selected from natural non-toxic and eco-friendly substances without any adverse effects on the environment. Figure shows the main steps in the green synthesis of metal nanoparticles.

Steps involved in the biosynthesis of nanoparticles [4]:

(Figure 1)

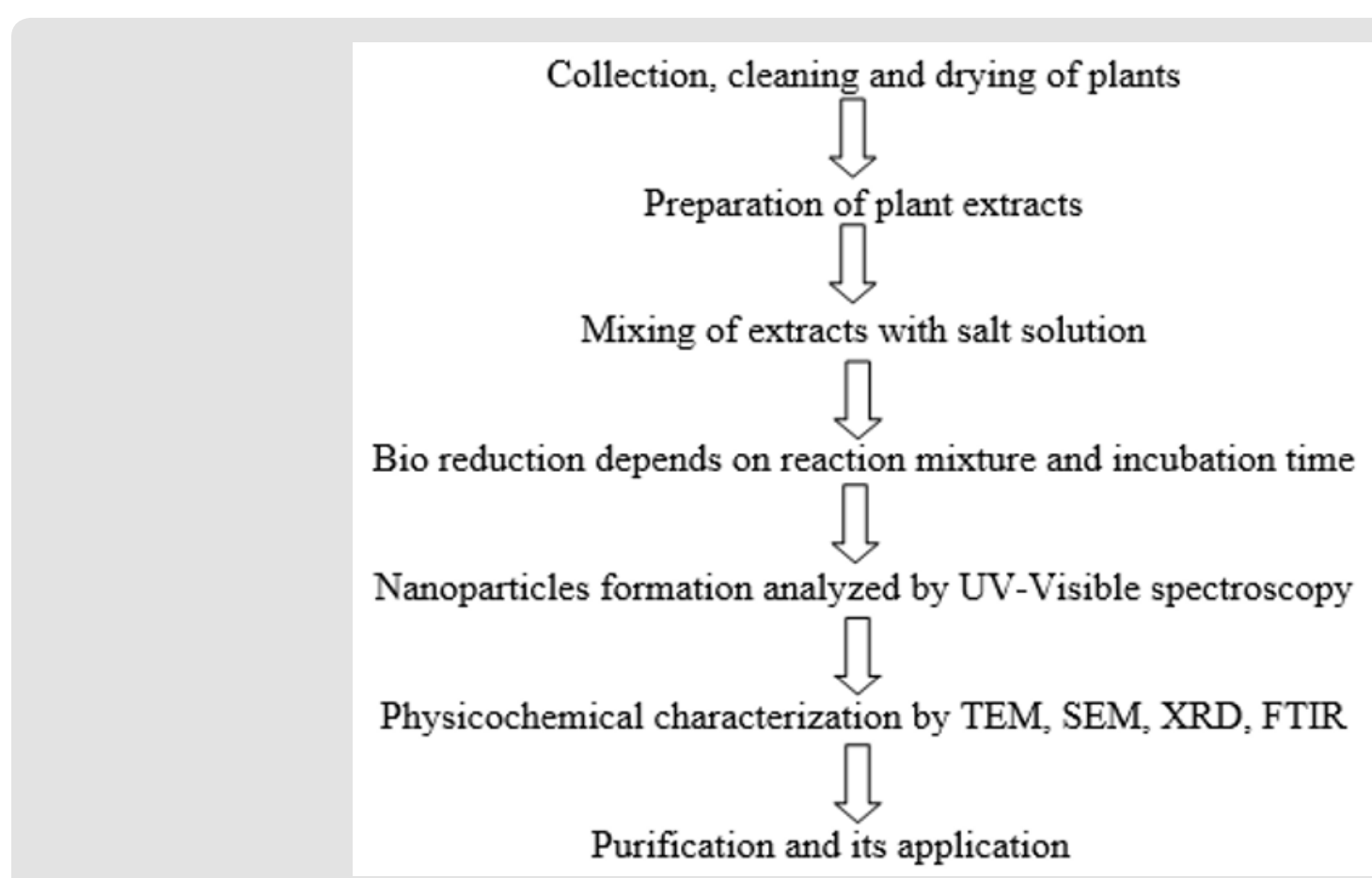

Figure 1.

\section{Methodology}

1. About 180 articles were collected from various databases including Google Scholars, ScienceDirect, Sci-Hub, PubMed, ResearchGate and studied.

2. From 180 articles, 100 articles were collected for green synthesis of antimicrobial study.

3. Finally, 20 plants were reviewed comprehensively.

\section{Result and Discussion}

(Tables $1 \&$ 2)

Many research papers reported the synthesis of silver nanoparticles using plant extracts such as Croton sparsiflorus (Ban tulasi) [1]; Chlorophytum borivilianum (Musli) [5]; Musa paradisiaca (Banana) [6]; Aloe vera [7]; Enteromorpha flexuosa (Green alga) [8]; salvinia molesta (Giant salvinia or exotic weed) [9]; Cissus quadrangularis (Veldt grape) [10]; Ficus benghalensis (Banyan) [11]; Azadirachta indica (Neem) [11]; Cocos nucifera (Coconut) [12]; Pithophora oedogonia (Green alga) [13]; Aegle marmelos (Bael) [14]; Dalbergia spinosa [15]; Lythrum salicaria (Purple loosestrife) [16]; Euphorbia confinalis (Spurge) [17]; Withania somnifera (Ashwagandha) [18]; Justicia adhatoda L. (Vasaka) [19]; Chrysanthemum indicum (Chandramallika) [20]; Taraxacum officinale (Dandelion) [21]; Phoenix dactylifera (Date palm) [22]. 
Table 1: Green synthesis of silver nanoparticles using plants extracts.

\begin{tabular}{|c|c|c|c|c|c|c|}
\hline $\begin{array}{l}\text { Plants name \& } \\
\text { Family }\end{array}$ & $\begin{array}{l}\text { Parts used/ } \\
\text { Extract }\end{array}$ & Microorganisms & Phytochemical compounds & $\begin{array}{l}\text { Metal } \\
\text { used }\end{array}$ & $\begin{array}{l}\text { Size }(\mathrm{nm}) \text { and } \\
\text { shape }\end{array}$ & Ref. \\
\hline $\begin{array}{l}\text { Croton sparsiflorus } \\
\text { (Ban tulasi); Euphor- } \\
\text { biaceae }\end{array}$ & Root & $\begin{array}{l}\text { Staphylococcus aureus }(19.62 \\
\text { mm), Klebsiella pneumonia } \\
(10.14 \mathrm{~mm}), \text { Pseudomonas } \\
\text { aureginosa }(9.12 \mathrm{~mm})\end{array}$ & $\begin{array}{l}\text { glycosides, saponins, tannins, flavo- } \\
\text { noids, terpenoids, alkaloids, crotspar- } \\
\text { ine, phenolics, N-methyl-crotsprine, } \\
\text { and N, O- dimethyl crotspar- } \\
\text { ine,Crotsparinine and N-methyl } \\
\text { crotsparinine }\end{array}$ & $\mathrm{Ag}$ & $\begin{array}{l}36.51-42.49 \mathrm{~nm} ; \\
\text { spherical }\end{array}$ & 1 \\
\hline $\begin{array}{l}\text { Chlorophytum } \\
\text { borivilianum (Musli); } \\
\text { Asparagaceae }\end{array}$ & Root & $\begin{array}{l}\text { Pseudomonas aeruginosa } \\
(15 \mathrm{~mm}), \text { Proteus mirabilis } \\
(15 \mathrm{~mm}), \text { Coagulase-positive } \\
\text { Staphylococcus }(13 \mathrm{~mm}), \text { En- } \\
\text { terococcus faecalis }(13 \mathrm{~mm})\end{array}$ & $\begin{array}{l}\text { saponins, flavones, terpenoids, } \\
\text { glycoside }\end{array}$ & Ag & $\begin{array}{l}\text { 30-50nm; spher- } \\
\text { ical }\end{array}$ & 5 \\
\hline $\begin{array}{l}\text { Musa paradisiacal } \\
\text { (Banana); Musaceae }\end{array}$ & Peel & $\begin{array}{c}\text { B. Subtilis }(12 \mathrm{~mm}) \text {, S.aureus } \\
(16 \mathrm{~mm}), \text { Pseudomonas aerugi- } \\
\text { nosa }(20 \mathrm{~mm}), \text { Escherichia coli } \\
(17 \mathrm{~mm})\end{array}$ & $\begin{array}{l}\text { lignin, hemicellulose, cellulose and } \\
\text { pectins }\end{array}$ & Ag & $\begin{array}{l}\text { 23.7nm; spherical } \\
\text { \& crystalline }\end{array}$ & 6 \\
\hline $\begin{array}{l}\text { Aloe vera; Asphodela- } \\
\text { ceae }\end{array}$ & Leaf & $\begin{array}{c}\text { Staphylococcus aureus } \\
(0.014 \mathrm{~mm}), \text { Escherichia coli } \\
(0.007 \mathrm{~mm})\end{array}$ & $\begin{array}{l}\text { Lignin, flavonoids, hemicellulose, pec- } \\
\text { tin, polyphenols, citric acid, ascorbic } \\
\text { acid, and acetic acid }\end{array}$ & Ag & 23nm; Spherical & 7 \\
\hline $\begin{array}{l}\text { Enteromorpha flex- } \\
\text { uosa (Green alga); } \\
\text { Ulvaceae }\end{array}$ & Seaweed & $\begin{array}{l}\text { B. subtilis }(18 \pm 0.8 \mathrm{~mm}), \text { B. } \\
\text { pumulis( } 19 \pm 1.2 \mathrm{~mm}), \text { E. faeca- } \\
\text { lis }(12 \pm 0.9 \mathrm{~mm}), \text { S. aureus }(14 \pm \\
0.7 \mathrm{~mm}), \text { S. epidermidis }(20 \pm \\
\text { 1.5mm), E. coli }(13 \pm 0.9 \mathrm{~mm}) \\
\text { K. pneumoniae }(10 \pm 0.4 \mathrm{~mm}), \\
\text { C. albicans }(14 \pm 0.8 \mathrm{~mm}), \text { S. } \\
\text { cerevisiae }(16 \pm 0.6 \mathrm{~mm})\end{array}$ & $\begin{array}{l}\text { carbohydrates, alkaloids, steroids, } \\
\text { phenols, saponins and flavonoids }\end{array}$ & Ag & 2-32nm; Circular & 8 \\
\hline $\begin{array}{c}\text { salvinia molesta (Gi- } \\
\text { ant salvinia or exotic } \\
\text { weed); Salviniaceae }\end{array}$ & Leaf & $\begin{array}{l}\text { Escherichia coli }(21 \mathrm{~mm}) \text { and } \\
\text { Staphylococcus aureus }(16 \mathrm{~mm})\end{array}$ & $\begin{array}{l}\text { phenol derivatives, proteins, reducing } \\
\text { sugars, flavonoids, and enzymes }\end{array}$ & Ag & $\begin{array}{l}12.46 \mathrm{~nm} \text {; Spher- } \\
\text { ical }\end{array}$ & 9 \\
\hline $\begin{array}{l}\text { Cissus quadrangu- } \\
\text { laris (Veldt grape); } \\
\text { Vitaceae }\end{array}$ & Stem & $\begin{array}{l}\text { Klebsiella planticola }(13 \mathrm{~mm}) \\
\text { Bacillus subtilis }(11 \mathrm{~mm})\end{array}$ & $\begin{array}{l}\text { alcohols, carboxylic acids, phenols, } \\
\text { amines and amides }\end{array}$ & $\mathrm{Ag}$ & $\begin{array}{l}\text { 37- } 44 \mathrm{~nm} \text {; rod, } \\
\text { spherical \& } \\
\text { triangle }\end{array}$ & 10 \\
\hline $\begin{array}{l}\text { Ficus benghalensis } \\
\text { (Banyan); Moraceae }\end{array}$ & Bark & $\begin{array}{c}\text { E. coli }(13 \mathrm{~mm}), \text { P. aerugino- } \\
\text { sa }(14 \mathrm{~mm}) \text { and V. cholera } \\
(14.1 \mathrm{~mm}) \text { and } B . \text { subtilis } \\
(13.8 \mathrm{~mm})\end{array}$ & $\begin{array}{l}\text { leucopelargonidin-3-0- } \alpha \text {-L rham- } \\
\text { noside, beta glucoside, leucocyni- } \\
\text { din-3-0- } \alpha \text {-D galactosyl cellobioside, } \\
\text { glucoside, pentatriacontan-5-one and } \\
\text { beta sitosterol- } \alpha \text {-D-glucose }\end{array}$ & Ag & $40 \mathrm{~nm}$ & 11 \\
\hline $\begin{array}{l}\text { Azadirachta indica } \\
\text { (Neem); Meliaceae }\end{array}$ & Bark & $\begin{array}{l}\text { E. coli }(13 \mathrm{~mm}), \text { P. aerugi- } \\
\text { nosa }(14 \mathrm{~mm}) \text { and V.cholera } \\
(14.1 \mathrm{~mm}) \text { and B. subtilis } \\
(13.8 \mathrm{~mm})\end{array}$ & $\begin{array}{c}\text { Nimbin, Nimbinin, Deacetyl nimbin, } \\
\text { Nimbinene, 6-Deacetyl nimbinene, } \\
\text { Nimbandiol, polysaccharides G1A, } \\
\text { G1B, G2A, and G3A, NB-2 peptidoglu- } \\
\text { can }\end{array}$ & Ag & $50 \mathrm{~nm}$ & 11 \\
\hline $\begin{array}{l}\text { Cocos nucifera (Coco- } \\
\text { nut); Arecaceae }\end{array}$ & Leaf & $\begin{array}{l}\text { Klebsiella pneumonia }(24 \\
\text { mm), Plesiomonas shigelloides } \\
(21 \mathrm{~mm}), \text { Vibrio alginolyticus } \\
(19 \mathrm{~mm}) \text { and Salmonella } \\
\text { paratyphi }(16 \mathrm{~mm}), \text { P. aerugi- } \\
\text { nosa }(14 \mathrm{~mm}), \text { Vibrio harveyi } \\
(14 \mathrm{~mm}), \text { Bacillus subtilis } \\
(14 \mathrm{~mm}), \text { E. coli }(12 \mathrm{~mm})\end{array}$ & $\begin{array}{l}\text { Tannin, alkaloids, carbohydrates, } \\
\text { terpenoids, saponins, phenolic com- } \\
\text { pounds and reducing sugar }\end{array}$ & Ag & $22 \mathrm{~nm}$; Spherical & 12 \\
\hline $\begin{array}{l}\text { Pithophora oedogo- } \\
\text { nia (Mont.) Wittrock } \\
\text { (Green alga); Pitho- } \\
\text { phoraceae }\end{array}$ & Green algae & $\begin{array}{l}\text { E. coli, Pseudomonas aerugi- } \\
\text { nosa }(17.2 \mathrm{~mm}), \text { E. coli }(16.8 \\
\text { mm), Vibrio cholera, Shigella } \\
\text { flexneri, Bacillus subtilis, } \\
\text { Staphylococcus aureus, and } \\
\text { Micrococcus luteus }\end{array}$ & $\begin{array}{l}\text { Carbohydrates, steroids,saponins, } \\
\text { tannins, and protein }\end{array}$ & Ag & $\begin{array}{l}\text { 34.03nm; cubic \& } \\
\text { hexagonal }\end{array}$ & 13 \\
\hline $\begin{array}{l}\text { Aegle marmelos } \\
\text { (Bael); Rutaceae }\end{array}$ & Leaf \& fruit & $\begin{array}{c}\text { B. Cereus }(19.25 \pm 0.19 \mathrm{~mm}) \text {, } \\
\text { P. aeruginosa }(16.50 \pm \\
0.30 \mathrm{~mm}), \text { S. dysentriae }(15.90 \\
\pm 0.85 \mathrm{~mm}), \text { E. Coli }(15.15 \pm \\
0.62 \mathrm{~mm}), \text { S. Typhi }(14.50 \pm \\
0.70 \mathrm{~mm}), \text { Y. Pestis }(14.65 \pm \\
0.38 \mathrm{~mm}), \text { S. Aureus }(15.22 \pm \\
\text { 0.52mm) }\end{array}$ & $\begin{array}{c}\text { carbohydrates, steroids, soluble } \\
\text { starch, tannins, terpenoids, flavonoids, } \\
\text { saponin, and alkaloid }\end{array}$ & $\mathrm{Ag}$ & $\begin{array}{l}159.76 \mathrm{~nm} \text { to } \\
181.36 \mathrm{~nm} \text {; Crys- } \\
\text { talline \& spherical }\end{array}$ & 14 \\
\hline
\end{tabular}




\begin{tabular}{|c|c|c|c|c|c|c|}
\hline $\begin{array}{l}\text { Dalbergia spinosa; } \\
\text { Fabaceae }\end{array}$ & Leaf & $\begin{array}{c}\text { Bacillus subtilis }(23 \mathrm{~mm}), \text { Pseu- } \\
\text { domonas aeruginosa }(19 \mathrm{~mm}), \\
\text { Staphylococcus aureus } \\
(21 \mathrm{~mm}) \text { and } \text { E. coli }(28 \mathrm{~mm})\end{array}$ & $\begin{array}{l}\text { flavonoids, isoflavonoids, neoflavo- } \\
\text { noids, steroids, terpenoids }\end{array}$ & $\mathrm{Ag}$ & $\begin{array}{l}18 \pm 4 \mathrm{~nm} \text {; spher- } \\
\quad \text { ical }\end{array}$ & 15 \\
\hline $\begin{array}{l}\text { Lythrum salicaria } \\
\text { (Purple loosestrife); } \\
\text { Lythraceae }\end{array}$ & Aerial & $\begin{array}{l}\text { S.aureus }(17.62 \pm 0.205 \mathrm{~mm}) \\
\text { and E.coli }(14.54 \pm 0.234 \mathrm{~mm})\end{array}$ & $\begin{array}{l}\text { phenolics, terpenoids, flavones, and } \\
\text { polysaccharides }\end{array}$ & $\mathrm{Ag}$ & $\begin{array}{l}\text { 45to65nm; } \\
\text { spherical }\end{array}$ & 16 \\
\hline $\begin{array}{l}\text { Euphorbia confinalis } \\
\text { (Spurge); Euphor- } \\
\text { biaceae }\end{array}$ & Stems & $\begin{array}{l}\text { E. coli }(11 \mathrm{~mm}) \text { and S. aureus } \\
\text { (13 mm) }\end{array}$ & $\begin{array}{l}\text { oils, keto steroids, glycosides, cou- } \\
\text { roupitine, indirubin, isatin, phenolic } \\
\text { compounds, flavonoids, and ter- } \\
\text { penoids }\end{array}$ & $\mathrm{Ag}$ & $\begin{array}{l}\text { 12nm-18nm; } \\
\text { spherical }\end{array}$ & 17 \\
\hline $\begin{array}{l}\text { Withania somnifera } \\
\text { (Ashwagandha); } \\
\text { Solanaceae }\end{array}$ & $\begin{array}{l}\text { Leaves, fruits, } \\
\text { and roots }\end{array}$ & $\begin{array}{c}\text { P. vulgaris, E. coli, and A. } \\
\text { tumefaciens, Candida albicans, } \\
\text { Proteus vulgaris }\end{array}$ & $\begin{array}{l}\text { Withanolide, Catechin, p-coumaric } \\
\text { acid, Luteolin-7-glucoside }\end{array}$ & $\mathrm{Ag}$ & $\begin{array}{l}70 \text { \& } 110 \mathrm{~nm} ; \\
\text { spherical }\end{array}$ & 18 \\
\hline $\begin{array}{l}\text { Justicia adhatoda } \\
\text { (Vasaka); Acantha- } \\
\text { ceae }\end{array}$ & Leaf & $\begin{array}{c}\text { Pseudomonas aeruginosa (7-9 } \\
\mathrm{mm})\end{array}$ & $\begin{array}{l}\text { vasicine, vasicinine, vasicinol, } \\
\text { vasicinone, essential oil, maiontone, } \\
\text { deoxyvasicinone, vasicol, glucoside } \\
\text { sitosterol, kaempferol. alkaloids }\end{array}$ & $\mathrm{Ag}$ & $20 \mathrm{~nm}$; spherical & 19 \\
\hline $\begin{array}{l}\text { Chrysanthemum in- } \\
\text { dicum (Chandramal- } \\
\text { lika); Sapotaceae }\end{array}$ & Flower & $\begin{array}{c}\text { Staphylococcus aureus } \\
(8.33 \pm 0.57 \mathrm{~mm}), \text { E. coli } \\
(13.00 \pm 0.90 \mathrm{~mm}), \text { Klebsiella } \\
\text { pneumonia }(19.10 \pm 0.50 \mathrm{~mm}) \\
\text { Pseudomonas aeruginosa } \\
(09.60 \pm 0.51 \mathrm{~mm})\end{array}$ & $\begin{array}{l}\text { Tannins, fllavonoids, proteins, glyco- } \\
\text { sides reducing sugars }\end{array}$ & $\mathrm{Ag}$ & $\begin{array}{l}37.71-71.99 \mathrm{~nm} \\
\text { spherical and } \\
\text { hexagonal }\end{array}$ & 20 \\
\hline $\begin{array}{l}\text { Taraxacum officinale } \\
\text { (Dandelion); Aster- } \\
\text { aceae }\end{array}$ & Flower & $\begin{array}{c}\text { Enterococcus faecalis (10- } \\
0.50 \mathrm{~mm}), \text { Pseudomonas } \\
\text { aeruginosa }(11-0.76 \mathrm{~mm}) .\end{array}$ & $\begin{array}{l}\text { Flavonoids, phenols, proteins, ter- } \\
\text { penoids, alkaloids }\end{array}$ & $\mathrm{Ag}$ & $\begin{array}{l}545 \mathrm{~nm} \pm 5 \mathrm{~nm} ; \\
\text { crystalline and } \\
\text { spherical }\end{array}$ & 21 \\
\hline $\begin{array}{l}\text { Phoenix dactylifera } \\
\text { (Date palm); Areca- } \\
\text { ceae }\end{array}$ & Seed & $\begin{array}{l}24 \mathrm{~mm}) \text { at highest concentra- } \\
\text { tions ( } 500 \mu \mathrm{g} / \mathrm{ml}),(11 \mathrm{~mm}) \text { at } \\
\text { lowest concentrations ( } 7.8 \mu \mathrm{g} / \\
\text { ml) against Methicillin-resis- } \\
\text { tant } S \text {. aureus }\end{array}$ & $\begin{array}{l}\text { phenolics, flavonoids, polyphenols, } \\
\text { aldehydes, carboxylic acids, saponin, } \\
\text { anthraquinone, terpenoids, proteins }\end{array}$ & $\mathrm{Ag}$ & $\begin{array}{l}\text { 14-30nm; spher- } \\
\text { ical }\end{array}$ & 22 \\
\hline
\end{tabular}

Table 2: Comparative study between plant extracts \& nanoparticles using plant extracts.

\begin{tabular}{|c|c|c|c|c|}
\hline \multirow{2}{*}{ Plants name } & \multirow{2}{*}{ Microorganisms } & \multicolumn{2}{|c|}{ Antimicrobial potential } & \multirow{2}{*}{ Ref. } \\
\hline & & Plant extracts (ZOI in mm) & Ag Nps (ZOI in mm) & \\
\hline \multirow{4}{*}{ Chlorophytum borivilianum } & P. aeruginosa & $6 \mathrm{~mm}$ & $15 \mathrm{~mm}$ & 5 \\
\hline & Proteus mirabilis & $6 \mathrm{~mm}$ & $15 \mathrm{~mm}$ & \\
\hline & Coagulase-positive Staphylococcus & $6 \mathrm{~mm}$ & $13 \mathrm{~mm}$ & \\
\hline & E. faecalis & $6 \mathrm{~mm}$ & $13 \mathrm{~mm}$ & \\
\hline \multirow{4}{*}{ Musa paradisiaca (Banana) } & B. subtilis & $0 \mathrm{~mm}$ & $12 \mathrm{~mm}$ & 6 \\
\hline & S.aureus & $0 \mathrm{~mm}$ & $16 \mathrm{~mm}$ & \\
\hline & P. aeruginosa & $0 \mathrm{~mm}$ & $20 \mathrm{~mm}$ & \\
\hline & E. coli & $0 \mathrm{~mm}$ & $17 \mathrm{~mm}$ & \\
\hline \multirow{9}{*}{ Enteromorpha flexuosa } & B. subtilis & $15 \pm 0.9 \mathrm{~mm}$ & $18 \pm 0.8 \mathrm{~mm}$ & 8 \\
\hline & B. pumulis & $16 \pm 1.0 \mathrm{~mm}$ & $19 \pm 1.2 \mathrm{~mm}$ & \\
\hline & E. faecalis & $11 \pm 0.7 \mathrm{~mm}$ & $12 \pm 0.9 \mathrm{~mm}$ & \\
\hline & S. aureus & $12 \pm 0.6 \mathrm{~mm}$ & $14 \pm 0.7 \mathrm{~mm}$ & \\
\hline & S. epidermidis & $18 \pm 1.2 \mathrm{~mm}$ & $20 \pm 1.5 \mathrm{~mm}$ & \\
\hline & E. coli & $11 \pm 0.9 \mathrm{~mm}$ & $13 \pm 0.9 \mathrm{mmm}$ & \\
\hline & K. pneumoniae & $10 \pm 0.5 \mathrm{~mm}$ & $10 \pm 0.4 \mathrm{~mm}$ & \\
\hline & C. albicans & $12 \pm 0.8 \mathrm{~mm}$ & $14 \pm 0.8 \mathrm{~mm}$ & \\
\hline & S. cerevisiae & $12 \pm 0.8 \mathrm{~mm}$ & $16 \pm 0.6 \mathrm{~mm}$ & \\
\hline
\end{tabular}




\begin{tabular}{|c|c|c|c|c|}
\hline \multirow{8}{*}{ Cocos nucifera } & Vibrio alginolyticus & $12 \mathrm{~mm}$ & $19 \mathrm{~mm}$ & 12 \\
\hline & P. shigelloides & $9 \mathrm{~mm}$ & $21 \mathrm{~mm}$ & \\
\hline & K. pneumoniae & $12 \mathrm{~mm}$ & $24 \mathrm{~mm}$ & \\
\hline & Salmonella paratyphi & $15 \mathrm{~mm}$ & $16 \mathrm{~mm}$ & \\
\hline & P. aeruginosa & $10 \mathrm{~mm}$ & $14 \mathrm{~mm}$ & \\
\hline & Vibrio harveyi & $13 \mathrm{~mm}$ & $14 \mathrm{~mm}$ & \\
\hline & Bacillus subtilis & $12 \mathrm{~mm}$ & $14 \mathrm{~mm}$ & \\
\hline & E. coli & $10 \mathrm{~mm}$ & $12 \mathrm{~mm}$ & \\
\hline \multirow{3}{*}{ Aegle marmelos } & E. coli & $9.0 \pm 0.15 \mathrm{~mm}$ & $15.15 \pm 0.62 \mathrm{~mm}$ & 14 \\
\hline & S. typhi & $9.16 \pm 0.54 \mathrm{~mm}$ & $15.22 \pm 0.52 \mathrm{~mm}$ & \\
\hline & B. cereus & $10.15 \pm 0.62 \mathrm{~mm}$ & $19.25 \pm 0.19 \mathrm{~mm}$ & \\
\hline \multirow{4}{*}{ Dalbergia spinosa } & S. aureus & $17 \mathrm{~mm}$ & $21 \mathrm{~mm}$ & 15 \\
\hline & E. coli & $19 \mathrm{~mm}$ & $28 \mathrm{~mm}$ & \\
\hline & B. subtilis & $16 \mathrm{~mm}$ & $23 \mathrm{~mm}$ & \\
\hline & P. aeruginosa & $14 \mathrm{~mm}$ & $19 \mathrm{~mm}$ & \\
\hline \multirow{2}{*}{ Euphorbia confinalis } & Staphylococcus aureus & $2 \mathrm{~mm}$ & $13 \mathrm{~mm}$ & 17 \\
\hline & Escherichia coli & $2 \mathrm{~mm}$ & $11 \mathrm{~mm}$ & \\
\hline \multirow{2}{*}{ Taraxacum officinale } & E. Faecalis & $10 \pm 0.50 \mathrm{~mm}$ & $12 \pm 0.27 \mathrm{~mm}$ & 21 \\
\hline & P. aeruginosa & $11 \pm 0.76 \mathrm{~mm}$ & $14 \pm 0.90 \mathrm{~mm}$ & \\
\hline Phoenix dactylifera & $\begin{array}{c}\text { Methicillin-resistant Staphylococcus } \\
\text { aureus (MRSA) }\end{array}$ & $9 \mathrm{~mm}$ & $11 \mathrm{~mm}$ & 22 \\
\hline
\end{tabular}

The nanoparticles were engineered via reduction of silver nitrate $\left(\mathrm{AgNO}_{3}\right)$ solution with aqueous root extract of C. borivilianum at $50{ }^{\circ} \mathrm{C}$. The root extract of Chlorophytum borivilianum as capping agent with an average diameter of $30-50 \mathrm{~nm}$. The formation of NPs is analyzed by UV-Vis spectroscopy, distinctive phases and morphology are confirmed by using XRD, SEM, TEM, and FTIR is used to identify the biomolecules which are responsible for reduction and stabilization of NPs. These biologically synthesized AgNPs were found to highly toxic against some clinically pathogenic bacteria such as coagulase positive Staphylococcus sp., Enterococcus faecalis, Pseudomonas species, and Proteus mirabilis with reference to commercially available antibiotics [5]. The biosynthesized silver nanoparticles using banana peel extract were characterized by UV-Vis spectrophotometer, XRD, DLS, TEM, SEM, DLS, and FTIR; they are crystalline, uniform, spherical and monodispersed nanoparticles with average particle size of $23.7 \mathrm{~nm}$. Synthesized silver nanoparticles revealed good antimicrobial activity against B. subtilis, S.aureus, E. coli [6]. The phytochemical compounds like Lignin, hemicellulose, pectin, flavonoids, polyphenols, ascorbic acid, citric acid, and acetic acid of Aloe vera (family: Asphodelaceae) as reducing agent showed $23 \mathrm{~nm}$ size $\mathrm{nps}$ with spherical shape [7].

Circular shape of AgNps within the range of 2-32nm were produced by using the seaweed extract of Enteromorpha flexuosa plant and showed more antibacterial activity than plant extract against B. subtilis, B. pumulis, E. faecalis, S. aureus, S. epidermidis, E. coli, K. pneumoniae, C. albicans, $S$. cerevisiae. The reduced silver nanoparticles were characterized by UV-vis spectrophotometer,
EDS, XRD and TEM [8]. FESEM, EDX, HRTEM, AFM and XRD analysis showed that most of AgNPs of salvinia molesta extracts were spherical in shape with average size distribution of $12.46 \mathrm{~nm}$ having face centered cubic (fcc) crystal lattice. Antibacterial studies reviled the better efficacy of AgNPs against Gram negative bacteria as compared to Gram positive bacteria [9]. Cissus quadrangularis stem extracts showed mostly spherical and some rod and triangle shapes with sizes ranging from 37 to $44 \mathrm{~nm}$, which were characterized by SEM. FTIR shows that the functional groups are carboxyl, amine, and phenolic compounds of stem extract which are involved in the reduction of silver ions. Thus, synthesized silver nanoparticles show more antibacterial activity against Klebsiella planticola and Bacillus subtilis, which was analyzed by disc diffusion method [10].

Ag-Nps were synthesized by using phytochemical compounds (eucopelargonidin-3-0- $\alpha$-L rhamnoside, leucocynidin-3-0- $\alpha$-D galactosyl cellobioside, glucoside, beta glucoside, pentatriacontan5 -one and beta sitosterol- $\alpha$-D-glucose) of Ficus benghalensis (family: Moraceae) with size 40nm and analyzed by UV-spectrophotometer, DLS, Fe-SEM, AFM and ATR-FTIR [11]. Various phytochemicals like nimbin, nimbinin, deacetyl nimbin, nimbinene, 6-deacetyl nimbinene, nimbandiol, polysaccharides G1A, G1B, G2A, and G3A, and NB-2 peptidoglucan were synthesized from the bark extract of Azadirachta indica and used in the synthesis of Ag-Nps with $50 \mathrm{~nm}$ size and showed antimicrobial activity against B. subtilis, E. coli, P. aeruginosa and V. cholerae [11]. The synthesized silver nanoparticles showed maximum activity by using leaf extract of coconut tree against K. pneumoniae, P. shigelloides, V. Alginolyticus, 
Salmonella paratyphi and Bacillus subtilis and produced 22nm size with spherical shape nanoparticles. The synthesized nanoparticles were characterized by UV-visible spectroscope, FTIR and TEM analysis [12].

Pithophora oedogonia (Mont.) Wittrock extract as a reducing agent can effectively produced $34.03 \mathrm{~nm}$ cubical and hexagonal shape silver nanoparticles. Characterization of synthesized silver nanoparticles was carried out by UV-vis spectroscopy, FTIR, DLS and SEM equipped with EDS [13]. Phytochemical analysis of methanolic extract of Aegle marmelos revealed the presence of tannins, saponins, steroids, alkaloids, flavonoids, and glycosides. Agar well diffusion method was used for determining antimicrobial activity of AgNPs. AgNPs synthesized using Aegle marmelos methanolic extract, characterized by UV-Visible spectroscopy, atomic force microscopy, dynamic light scattering, and X-ray diffraction showed size ranged between 159 and $181 \mathrm{~nm}$. Evaluation of the antimicrobial potential of green synthesized AgNPs recorded the highest inhibitory activity against $B$. cereus $(19.25 \pm 0.19 \mathrm{~mm}$ ) followed by P. aeruginosa (16.50 $\pm 0.30 \mathrm{~mm}$ ) and $S$. dysentriae [14]. Phytochemical compounds like flavonoids, isoflavonoids, neoflavonoids, steroids, terpenoids of leaf extract of Dalbergia spinosa used in the synthesis of Ag-Nps (18 \pm $4 \mathrm{~nm}$; spherical) and showed more activity against Bacillus subtilis, Pseudomonas aeruginosa, Staphylococcus aureus, and Escherichia coli than plant extract [15].

Lythrum salicaria extract was used as a reducing agent as well as a capping agent. Formation of the spherical AgNPs ranging between 45 and $65 \mathrm{~nm}$ was proved by UV-Vis spectroscopy, transmission electron microscopy (TEM), and dynamic light scattering (DLS). Biomaterials supported AgNPs were characterized and compared for their morphological, thermal, release, and antimicrobial properties [16]. The stem extract of Euphorbia confinalis from Euphorbiaceae family showed the formation of nanoparticles of spherical shaped with a size of $12-18 \mathrm{~nm}$. The synthesized silver nanoparticles showed maximum activity against E. coli, S. aureus and analyzed by UV-Vis, SEM, TEM, and FTIR [17]. The phytochemical compounds catechin, p-coumaric acid, luteolin-7glucoside, and a nonidentified withanolide derivative present in the Withania somnifera (Ashwagandha) aqueous leaf extract showed the formation of nanoparticles of spherical shaped with a size of 70 \& 110nm [18]. AgNPs were synthesized by reduction of AgNO3 solution using extract of Vasaka as capping agent and observed the spherical shaped nanoparticles and the average particle size is 20 $\mathrm{nm}$ in the range of 5-50 $\mathrm{nm}$.

The biosynthesized silver nanoparticles were characterized by UV-Vis spectroscopy and TEM analysis. The antibacterial activity of these nanoparticles against Pseudomonas aeruginosa was measured by disc diffusion method, agar cup assay and serial dilution turbidity measurement assay [19]. The phytochemical screen ing of Chrysanthemum indicum revealed the presence of flavonoids, terpenoids, and glycosides, suggesting that these compounds act as reducing and stabilizing agents. The spherical and hexagonal Ag-NPs were also synthesized by using Chrysanthemum indicum flower extract with an average particle size from 37.71$71.99 \mathrm{~nm}$ and characterized by using UV-Vis spectroscopy, XRD, TEM, and EDX. The antimicrobial effect of the synthesized AgNPs revealed a significant effect against the bacteria Klebsiella pneumonia, Escherichia coli, and Pseudomonas aeruginosa [20]. The biosynthesis of silver nanoparticles using Taraxacum officinale floral extract showed the formation of nanoparticles of spherical shaped with a size of $545 \mathrm{~nm} \pm 5 \mathrm{~nm}$ ) upon addition of $1 \mathrm{mM}$ silver nitrate. AgNPs synthesized from floral extract of T.officinale showed good antibacterial activity against selected pathogns such as Enterococcus faecalis and Pseudomonas aeruginosa by disc diffusion assay [21].

The spherical-shaped AgNPs were synthesized by using Phoenix dactylifera seed extract as stabilizing agent and characteristics of particles were studied by using UV-Vis spectroscopy, SEM, HR-TEM, and DLS. The antibacterial activities were found to be increased with the increasing concentration of AgNPs. The zone of inhibition was greater $(24 \mathrm{~mm})$ at highest concentrations $(500 \mu \mathrm{g} / \mathrm{ml})$ of AgNPs, while smaller $(11 \mathrm{~mm})$ at lowest concentrations $(7.8 \mu \mathrm{g} / \mathrm{ml})$ [22]. The silver nanoparticles (AgNPs) synthesized using hot water olive leaf extracts as reducing and stabilizing agent is evaluated for antibacterial activity against drug resistant bacterial isolates. The silver nanoparticles were with an average size of 20-25 nm and mostly spherical.

\section{Conclusion}

In summary, it is concluded that during the last decade many efforts have been made for the development of green synthesis. Green synthesis gives headway over chemical and physical methods as it is cost-effective, eco-accommodating and effectively scaled up for large-scale synthesis. Production of NPs using extracts from natural substances is emerging as an important area in nanotechnology. Plants are having broad range of phytochemicals like alkaloids, terpenoids, phenols, flavonoids, tannins and quinines etc. which can mediate the synthesis of nanoparticles. It was shown that a variety of plant extracts have been used to efficiently synthesize metal nanoparticles for green synthesis, but the metal nanoparticles produced by plants are more stable and bioactive in comparison with those produced by plant extracts. The findings of this study suggest that the nps synthesized by plant extracts exhibited good antimicrobial activity against bacterial pathogens which indicates the immense potential as effective antimicrobial agents that can be used in various modern medicines. Natural sources have the potential to reduce silver ions into AgNPs.

It is understood that the variety of natural compounds that are present in plant extracts can act as both reducing and stabilizing agents for synthesis of AgNPs. Plants mediated AgNPs are stable 
due to the presence of natural capping agents such as proteins which prevent the particles from aggregation. Green synthesis of AgNPs using plant extracts has several advantages such as ecofriendliness, biocompatibility and cost-effectiveness. It is concluded that due to these unique properties, AgNPs will have a key role in many of the nanotechnology-based processes.

\section{References}

1. Joshi P, Joy H, Vyas P (2016) Green Synthesis of Silver Nanoparticle Using Plant Root Extract of Croton sparsiflorus and their Antimicrobial Activity. International Journal of Science and Research 5(12).

2. Mohammadlou M, Maghsoudi H, and Jafarizadeh-Malmiri H (2016) A review on green silver nanoparticles based on plants: Synthesis, potential applications and eco-friendly approach. International Food Research Journal 23(2): 446-463.

3. Gupta M, Singh RT, Mishra RK (2020) Factors Affecting Biosynthesis of Green Nanoparticles. Our Heritage 68(30): 10530-10555.

4. Kavitha KS, Baker S, Rakshith D, Kavitha HU, Rao HCY, et al. (2013) Plants as Green Source towards Synthesis of Nanoparticles. International Research Journal of Biological Sciences 2(6): 66-76.

5. Singh S, Vyas R, Chaturvedi A, Sisodia R (2018) Rapid phytosynthesis of silver nanoparticles using Chlorophytum borivilianum root extract and its antimicrobial activity. Journal of Pharmacognosy and Phytochemistry 7(5): 1738-1744

6. Ibrahim HMM (2015) Green synthesis and characterization of silver nanoparticles using banana peel extract and their antimicrobial activity against representative microorganisms. Journal of Radiation Research and Applied Sciences 8(3): 265-275.

7. Ahmadi O, Malmiri HJ, Jodeiri N (2018) Eco-friendly microwaveenhanced green synthesis of silver nanoparticles using Aloe vera leaf extract and their physico-chemical and antibacterial studies. Green Processing and Synthesis 7(3): 231-240.

8. Yousefzadi M, Rahimi Z, Ghafori V (2014) The green synthesis, characterization and antimicrobial activities of silver nanoparticles synthesized from green alga Enteromorpha flexuosa (wulfen) J Agardh. Materials Letters 137: 1-4.

9. Verma DK, Hasan SH, Banik RM (2016) Photo-catalyzed and phytomediated rapid green synthesis of silver nanoparticles using herbal extract of salvinia molesta and its antimicrobial efficacy. Journal of Photochemistry and Photobiology B: Biology 155: 51-59.

10. Vanaja M, Gnanajobitha G, Paulkumar K, Rajeshkumar S, Malarkodi C, et al. (2013) Phytosynthesis of silver nanoparticles by Cissus quadrangularis: influence of physicochemical factors. Journal of Nanostructure in Chemistry 3(1).

11. Nayak D, Ashe S, Rauta PR, Kumari M, Nayak B (2016) Bark extractmediated green synthesis of silver nanoparticles: Evaluation of antimicrobial activity and antiproliferative response against osteosarcoma. Materials Science and Engineering: C 58: 44-52.

12. Mariselvam R, Ranjitsingh AJA, Nanthini AUR, Kalirajan K, Padmalatha C, et al. (2014) Green synthesis of silver nanoparticles from the extract of the inflorescence of Cocos nucifera (Family: Arecaceae) for enhanced antibacterial activity. Spectrochimica Acta Part A: Molecular and Biomolecular Spectroscopy 129: 537-541.

13. Sinha SN, Paul D, Halder N, Sengupta D, Patra SK (2014) Green synthesis of silver nanoparticles using fresh water green alga Pithophora oedogonia (Mont.) Wittrock and evaluation of their antibacterial activity. Applied Nanoscience 5(6): 703-709.

14. Devi M, Devi S, Sharma V, Rana N, Bhatia RK, et al. (2020) Green synthesis of silver nanoparticles using methanolic fruit extract of Aegle marmelos and their antimicrobial potential against human bacterial pathogens. Journal of Traditional and Complementary Medicine 10(2): 158-165.

15. Muniyappan N, Nagarajan NS (2014) Green synthesis of silver nanoparticles with Dalbergia spinosa leaves and their applications in biological and catalytic activities. Process Biochemistry 49(6): 10541061.

16. Mohammadalinejhad S, Almasi H, Esmaiili M (2019) Simultaneous green synthesis and in-situ impregnation of silver nanoparticles into organic nanofibers by Lythrum salicaria extract: Morphological, thermal, antimicrobial and release properties. Materials Science and Engineering: C 105: 110115.

17. Muchanyereyi NM, Muchenje N, Nyoni S, Shumba M, Mupa M, et al. (2017) Green Synthesis of Silver Nanoparticles Using Euphorbia Confinalis Stem Extract, Characterization and Evaluation of Antimicrobial Activity. Journal of Nanomaterials \& Molecular Nanotechnology 6(3).

18. Marslin G, Selvakesavan RK, Franklin G, Sarmento B, Dias AC (2015) Antimicrobial activity of cream incorporated with silver nanoparticles biosynthesized from Withania somnifera. International Journal of Nanomedicine pp. 5955.

19. Bose D, Chatterjee S (2015) Antibacterial Activity of Green Synthesized Silver Nanoparticles Using Vasaka (Justicia adhatoda L.) Leaf Extract. Indian Journal of Microbiology 55(2): 163-167.

20. Arokiyaraj S, Arasu MV, Vincent S, Prakash NU, Choi SH, et al. (2014) Rapid green synthesis of silver nanoparticles from Chrysanthemum indicum L. and its antibacterial and cytotoxic effects: an in vitro study. International journal of nanomedicine 9: 379-388.

21. Arokiyaraj S, Saravanan M, Vijayakumar B (2015) Green synthesis of Silver nanoparticles using aqueous extract of Taraxacum officinale and its antimicrobial activity. South Indian Journal of Biological Sciences 1(2): 115-118.

22. Ansari MA, Alzohairy MA (2018) One-Pot Facile Green Synthesis of Silver Nanoparticles Using Seed Extract of Phoenix dactylifera and Their Bactericidal Potential against MRSA. Evidence-Based Complementary and Alternative Medicine p. 1-9.
ISSN: 2574-1241

DOI: 10.26717/BJSTR.2021.34.005580

Md Shahidul Islam. Biomed J Sci \& Tech Res

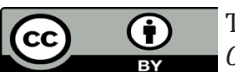

This work is licensed under Creative

Commons Attribution 4.0 License

Submission Link: https://biomedres.us/submit-manuscript.php

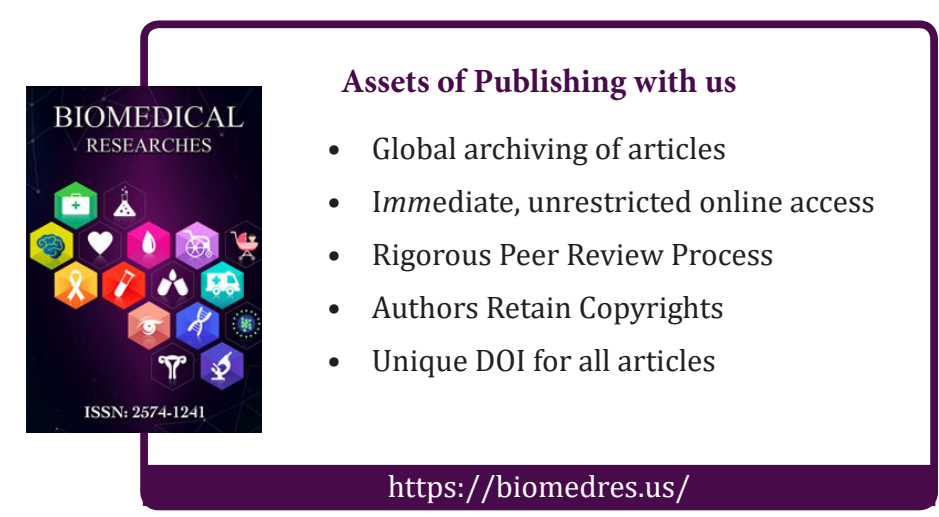

